\title{
Analysis of Energy- and SoC-Neutral Contracts for Frequency Regulation with Energy Storage
}

\author{
Dariush Fooladivanda \\ University of Waterloo \\ dfooladi@uwaterloo.ca
}

\author{
Catherine Rosenberg \\ University of Waterloo \\ cath@uwaterloo.ca
}

\author{
Siddharth Garg \\ New York University \\ sg175@nyu.edu
}

\begin{abstract}
We analyze two contractual frameworks for regulation services with battery-based energy storage: the SoCand energy-neutral contracts. Vis-a-vis the existing contractual framework, the new contracts constrain the operator to generate only SoC- and energy-neutral regulation signals within a contract duration. For both contracts, we propose algorithms to maximize the battery operator's reward during a single contract, and derive analytical expressions to quantify the reward over multiple contracts. Numerical results using Lead-Acid and Lithium-ion batteries provide several engineering insights.
\end{abstract}

\section{INTRODUCTION}

Electrical grid operators have the responsibility to maintain the balance between demand and supply at all times using services that operate at different time scales [1]. They typically predict the demand a day ahead, and schedule some slow-ramping generators for supplying electricity. Then, the target grid frequency is maintained by performing real-time adjustments using fast-ramping generators or flexible loads [1]. Another approach to provide regulation is to use energy storage technologies (EST) that are able to recycle energy and provide fast and accurate responses. This approach has been adopted by several operators including the California Independent System Operator (CAISO) [2]. However, despite their speed and accuracy, there are some challenges linked to the EST limited capacity, charging and discharging power limits, and self-discharge. We will refer to a regulation unit (RU) that uses an EST as a storage regulation unit (SRU).

The context of this study is the design and analysis of contractual frameworks for regulation services better suited for SRUs. In a contractual framework, an SRU and an operator agree on a set of parameters and constraints on the regulations signals that the operator can send to the SRU. To illustrate this, let us focus on the existing framework [9]. A contract lasts over $D$ units of time, consisting of $K$ time-slots of length $\delta$, i.e., $D=K \delta$. This contract has been negotiated a few units of time ahead of time. During the negotiation phase, the operator and the SRU agree on the downward and upward regulation parameters $r$ and $R(r \leq 0 \leq R$, in Watts). The SRU commits to provide any power in the range $[r, R]$ in response to regulation signals sent by the operator. The operator commits to generate a sequence of regulation signals $s_{k}$ so that $s_{k} \in[r, R]$ in each time-slot $k \in\{1, \ldots, K\}$. The SRU has to supply constant power $s_{k}$ during time-slot $k$ if $s_{k}$ is positive, and draw constant power $\left(-s_{k}\right)$ from the grid if it is negative. Therefore, the only constraint on regulation signals in the existing framework is to make sure that the signals will be in the range $[r, R]$ declared by the SRU.
The SRU is rewarded for its flexibility in terms of the upward and downward regulation parameters $R$ and $r$, respectively, and for the actual amount of energy that it supplies/draws during the contract. The amount of energy that the SRU supplies/draws is not known at the beginning of a contract. Therefore, the SRU will select its parameters $r$ and $R$ so as to maximize the fixed part of the reward (call it $f(R,|r|)$ ) while keeping the risk of a regulation failure close to zero ${ }^{1}$.

Under the existing framework, the SRU would declare its regulation parameters as a function of its state of charge (SoC) at the beginning of the contract. In the following, when we refer to the SoC of a battery, we refer to the energy stored in the battery. The SoC at the end of the contract will be highly dependent on the regulation signals sent during the contract duration, making it challenging for the SRU to estimate its reward over several succeeding contracts [9]. This uncertainty in determining the reward ${ }^{2}$ over multiple contracts makes the task of planning and operation difficult for an SRU [9].

The primary goal of this paper is to propose and analyze alternative contractual frameworks that reduce this uncertainty from the perspective of the SRU. Different contractual frameworks can potentially create different burdens on the operator side, but we focus on the SRU standpoint. In the first framework, the operator commits to generating a sequence of regulation signals so that the $\mathrm{SoC}$ at the end of a contract is the same as the $\mathrm{SoC}$ at the beginning, i.e., there is no uncertainty on the SoC at the end of a contract. We call it the $\mathrm{SoC}$ neutral contractual framework. In this contractual framework, the $S R U$ operation will be greatly simplified, and computing the reward over several successive contracts will be as easy as computing the reward over one single contract. While there are many benefits with receiving SoC-neutral regulation signals for SRUs, sending SoC-neutral regulation signals limits the operator's flexibility by adding more constraints on the kind of regulation signals that the operator can send to an SRU and requires a very accurate model of the EST operations.

To reduce the burden on operators while hopefully offering high rewards, we consider another framework which is inspired in the framework deployed by CAISO [8]. In this framework, called the energy-neutral contractual framework, the operator commits to generate a sequence of regulation signals that are energy-neutral, i.e., the energy supplied by the SRU during the contract is equal to the energy absorbed by the SRU. It is obvious that if the energy storage was

\footnotetext{
${ }^{1}$ There is a penalty to pay if the RU cannot honor its commitments in terms of the regulation parameters $R$ and $r$.

${ }^{2} \mathrm{We}$ only refer to the fixed part of the reward when we refer to reward.
} 
perfect (i.e., $100 \%$ efficiency and no leakage), a sequence of regulation signals that is of zero mean would be SoC-neutral. However, in the presence of losses, the SoC at the end of multiple successive contracts would differ from the initial SoC. Therefore, after a limited number of contracts, the SRU might need to put the SoC of its storage back at the preferred level by either purchasing (or selling) electricity [7] or participating in a pure downward (or upward) regulation service.

The specific technical contributions of this paper are:

1) We first study the SoC-neutral contractual framework. For a single contract, we propose an algorithm to compute the regulation parameters $R$ and $r$ that the SRU should declare, given its initial SoC, to maximize its reward while ensuring that the risk of regulation failure is zero. We show that to obtain the highest possible reward in consecutive contracts in the SoC-neutral framework, the SRU needs to put the initial SoC of its storage at half of the storage capacity regardless of the EST parameters (for instance, charging and discharging rates and efficiences).

2) We then study the energy-neutral framework, first in the context of a single contract (as above) and then propose algorithms to quantify, ahead of time, the reward that an SRU could obtain in successive contracts using analytical upper and lower bounds.

3) We apply our techniques to gain engineering insights on the use of Lead-Acid and Li-ion ESTs for regulation, and empirically compare the rewards obtained for different technologies and contractual frameworks. We show that an energy-neutral framework provides an excellent tradeoff in terms of gains for the SRU and ease of implementation for the operator.

We now discuss the related work before presenting the main technical contributions of this paper.

\section{LITERATURE BACKGROUND}

A comprehensive overview of the conventional regulation services is provided in [3]. Recent studies have shown that as an alternative to conventional RUs, ESTs can provide fast and accurate regulation services [4]-[5], although some practical challenges, for instance energy storage management [2], [6], still remain. Several studies have indicated that regulation failures, i.e., when an SRU is unable to fulfill its contractual obligations because it is either fully depleted or at capacity, are a major concern for SRUs.

To avoid regulation failures, operators have tried to treat SRUs differently by combining them with hydro power plants [1], or allowing SRUs to purchase (or sell) electricity [7]. However, these approaches are not always feasible, for instance it is not always possible to co-locate with a hydropower facility, and do not reduce the risk of regulation failure to zero since perfect estimation of the SoC in the future is impossible.

In 2005, CAISO developed a new algorithm, called $A C E$ smoothing [2], [8], in which the regulation signal is broken up into a slow moving component (computed using a rolling average) and the remaining fast component that has zero mean. The former, because it is slow moving, is better suited for conventional generators with slow response times. The fast moving component is best suited for SRUs because of their fast response times and since it helps SRUs to maintain their SoC closer to the preferred level in consecutive contracts.

In our previous work [9], we have focused on the existing contractual framework in which the operator only guarantees that the regulation signals will lie between $r$ and $R$, and provided algorithms to allow SRUs to optimize its reward over a single contract, and determine lower and upper bounds on the reward over several successive contracts. However, in the existing framework, there is no constraint on the operator to only provide SoC- or energy-neutral signals. We believe that the analytical study provided in this paper is the first of its kind that enables SRUs to maximize and estimate rewards for the energy-neutral and SoC-neutral contractual frameworks.

\section{SySTEM MODEL}

Consider an SRU that offers regulation services in a region whose power system is controlled by an independent system operator. We assume that the time is slotted in time-slot $(t s)$ of size $\delta$, and that the duration of each contract is $K$ time-slots, i.e., $D=K \delta$. At the beginning of a contract, the SRU selects its regulation parameters $R$ and $r$ so that it can always respond to the regulation signals without any failure. We assume that the operator always accepts the declared parameters $R$ and $r$.

\section{A. EST Model}

We assume that the SRU is using a non-ideal battery of size $B^{\prime}$ (Watt-hour), with charging efficiency $e_{c}$, discharging efficiency $e_{d}$, maximum charging and discharging power limits $\Delta_{c}$ and $\Delta_{d}$ (Watt), respectively, and depth of discharge (DoD) $\eta$. Hence, the available capacity of the battery is equal to $B=\eta B^{\prime}$. The energy stored in an energy storage decays exponentially with a certain time constant whose value depends on the storage technology. This time constant is more than one year for batteries [10]. In this study, we neglect the impact of self-discharge since we study regulation services over a period of time which is less than 24 hours.

To present the SoC evolution model for electrochemical batteries, let us focus on one contract, and let $b(k)$ denote the SoC of the storage at the end of $t s k$. During the contract, the SRU will receive a sequence of signals $\left\{s_{1}, \cdots, s_{K}\right\}$. The SRU has to supply constant power $s_{k}$ during time-slot $k$ if $s_{k}$ is positive, and draw constant power $\left(-s_{k}\right)$ from the grid if it is negative. Therefore, $b(k)$ evolves as follows:

$$
b(k)=b(k-1)-e_{d} \delta\left[s_{k}\right]^{+}+e_{c} \delta\left[-s_{k}\right]^{+} \quad \forall k \in \mathcal{K}
$$

where $b(0)=U$ is the initial SoC, $\mathcal{K}=\{1, \ldots, K\}$, and $[x]^{+}$ is equal to $x$ if $x \geq 0$; otherwise, it is zero. By convention, in this model, we have $e_{c} \leq 1 \leq e_{d}$.

\section{B. Frequency Regulation Market}

In a regulation service, the SRU is rewarded for its flexibility in terms of the regulation parameters $R$ and $r$, and for what it actually supplies/draws during the contract. To select the values of the downward and upward regulation parameters, we focus on the fixed part of the reward, and assume that, in contract $C_{n}$, the SRU is paid a fixed price $a_{n} \geq 0$ (resp. $b_{n} \geq$ $0)$ per Watt of upward regulation (resp. downward regulation) for the contract duration $D$. Therefore, the fixed part of the 
regulation reward in a contract $C_{n}$ with regulation parameters $R_{n}$ and $r_{n}$ is calculated by

$$
f\left(R_{n},\left|r_{n}\right|\right)=\left(a_{n} R_{n}+b_{n}\left|r_{n}\right|\right) D \quad \text { (in dollars). }
$$

Next, we propose and study the SoC-neutral and energyneutral contractual frameworks.

\section{SoC-Neutral Framework}

In the SoC-neutral contractual framework, the operator commits to generating a sequence of regulation signals $\left\{s_{k}\right\}$ so that the SoC at the end of the contract is the same as the SoC, $U$, at the beginning of the contract, i.e., the regulation signals obey the following constraints:

$$
\begin{aligned}
& e_{d} \sum_{k=1}^{K}\left[s_{k}\right]^{+}=e_{c} \sum_{k=1}^{K}\left[-s_{k}\right]^{+} \\
& r \leq s_{k} \leq R \quad k \in \mathcal{K}
\end{aligned}
$$

The SRU can provide any power in the range $[r, R]$ in response to the regulation signals $\left\{s_{k}\right\}$ if and only if the following constraints are satisfied:

$$
\begin{aligned}
& 0 \leq b(k) \leq B \quad \forall k \in \mathcal{K} \\
& {\left[-s_{k}\right]^{+} \leq \Delta_{c} \quad \forall k \in \mathcal{K}} \\
& {\left[s_{k}\right]^{+} \leq \Delta_{d} \quad \forall k \in \mathcal{K} .}
\end{aligned}
$$

The main constraint imposed on the regulation signals is (3). Using this information, the SRU chooses its parameters $R$ and $r$ so that it can respond to all feasible sequences of regulation signals $\left\{s_{k}\right\}$ without any failure, and its reward $f(R,|r|)$ is maximized. We first compute the optimal values of $R, r$, and $U$ for one single contract of duration $D=K \delta$. Then, we focus on $N$ successive contracts.

\section{A. Single Contract}

Consider one single contract in which the operator provides regulation signals to the SRU so that obey (3). Starting with an initial SoC $U$, we are interested in finding the $(R, r)$ pair with the largest reward for that $U$. Then, we find the $U$ that yields largest reward over all values of $U$. We will do so in three steps. In the first step, we characterize the worst-case sequences of regulation signals that an operator can send to the SRU for a given $(R, r)$. By "worst-case", we mean the sequences of regulation signals that would result in the highest or lowest SoC levels during a contract duration. Then, in the second step, we develop an algorithm to compute the pair $(\overline{\mathbf{R}}, \overline{\mathbf{r}})$ that maximizes the reward given $U$. In the third step, we will select the value of the initial SoC $U^{\star}$ for which the SRU obtains the highest possible reward.

STEP 1 : Given $(R, r)$, we first observe that the sequence that would generate the highest (resp. lowest) SoC during a contract duration consists of a series of upwards (resp. downwards) regulation signals, followed by a series of downwards (resp. upwards) regulation signals. We characterize these worst-case sequences below.

We start with the sequence of regulation signals, $\pi_{m}$, that results in the highest SoC level. The sequence starts with $q_{m}$ time-slots of regulation signal $r$, where

$$
q_{m}=\left\lceil\frac{(K-1) e_{d} R+e_{c} r}{\left(e_{c}|r|+e_{d} R\right)}\right\rceil
$$

while the last $p_{m}=\left(K-1-q_{m}\right)$ time-slots have regulation signal $R$. There is a single time-slot in between the two, i.e., the $\left(q_{m}+1\right)^{t h}$ time-slot, with regulation signal $\left(-w_{m}\right)\left(\frac{1}{e_{c}} \mathbf{1}_{\left\{w_{m}>0\right\}}+\frac{1}{e_{d}} \mathbf{1}_{\left\{w_{m} \leq 0\right\}}\right)$, where $w_{m}=\left(e_{d} p_{m} R+\right.$ $\left.e_{c} q_{m} r\right)$. The sequence that provides the lowest SoC level, $\pi_{M}$, is the same as above except that it occurs in reverse order.

Although we do not provide our formal proof for the worstcase sequences above due to space constraints, we do note that, intuitively, if the signals were continuous then the worst-case sequences would be either a sequence of positive signals (all equal to $R$ ) followed by a sequence of negative signals (all equal to $r$ ) or a sequence of negative signals (all equal to $r$ ) followed by a sequence of positive signals (all equal to $R$ ). Call these sequences triangular sequences. This is in fact the case when $\frac{(K-1) R e_{d}+e_{c} r}{\left(e_{c}|r| e_{d} R\right)}$ is an integer. However, if it is not an integer, the worst-case sequences will be either a sequence of positive signals (all equal to $R$ ) followed by a single time-slot where the signal is not $R$ or $r$, followed by a sequence of negative signals (all equal to $r$ ) or the reverse. We call these sequences trapezoidal sequences since there exists one single time-slot in which the signal is different from $r$ and $R$.

STEP 2 : For the pair $(R, r)$ to be feasible given an initial SoC $U$, the SoC should always remain in the range $[0, B]$ for the corresponding worst-case sequences $\pi_{m}$ and $\pi_{M}$ determined in Step 1. We use our characterization of the worst cast sequences as the basis of a simple algorithm to find the pair $(\overline{\mathbf{R}}, \overline{\mathbf{r}})$ with the largest reward. In this algorithm, we first fix the number of downward regulation signals which are equal to the downward regulation effort $r$ at $q_{m} \in\{1, \cdots, K-1\}$. For a trapezoidal sequence, the number of upward regulation signals which are equal to the upward regulation effort $R$ will be $p_{m}=K-1-$ $q_{m}$, while for a triangular sequence $p_{m}=K-q_{m}$. Finally, we compute the reward corresponding to each combination of $q_{m}$ and $p_{m}$, and select the pair $(\overline{\mathbf{R}}, \overline{\mathbf{r}})$ that maximizes the reward. The following result provides a simple tool to find the optimal values of the parameters $R$ and $r$ for a given initial SoC, $U$.

Lemma 1. Given $e_{c}, e_{d}, \Delta_{c}, \Delta_{d}, U, \delta, B$, the reward function $f(R,|r|)$, and with $q_{m} \in\{1, \cdots, K-2\}$ and $p_{m}=K-1-$ $q_{m}$, or $q_{m} \in\{1, \cdots, K-1\}$ and $p_{m}=K-q_{m}$, the pair $(\overline{\mathbf{R}}, \overline{\mathbf{r}})$ that maximizes the reward, can be computed by

$$
\begin{aligned}
& \overline{\mathbf{R}}\left(q_{m}, p_{m}\right) \triangleq \min \left\{\Delta_{d}, \frac{e_{c} q_{m}}{e_{d} p_{m}} \Delta_{c}, \frac{B-U}{e_{d} \delta p_{m}}, \frac{U}{e_{d} \delta p_{m}}\right\} \\
& \left|\overline{\mathbf{r}}\left(q_{m}, p_{m}\right)\right| \triangleq \min \left\{\Delta_{c}, \frac{e_{d} p_{m}}{e_{c} q_{m}} \Delta_{d}, \frac{B-U}{e_{c} \delta q_{m}}, \frac{U}{e_{c} \delta q_{m}}\right\} .
\end{aligned}
$$

A formal description of the proposed algorithm to compute the optimal values of $R$ and $r$ is provided in Algorithm 1 . Next, we select the value of the initial SoC $U^{\star}$ for which the SRU obtains the highest possible reward.

STEP 3 : Given a set of parameters $B, K, \delta, \Delta_{c}, \Delta_{d}, e_{d}$, and $e_{c}$, different values of the initial SoC $U$ can result in different rewards. Let $\mathcal{U}^{\star}$ be the (set of) initial SoCs for which the SRU obtains the maximum reward. The following result shows that $\frac{B}{2} \in \mathcal{U}^{\star}$ irrespective of the values of the regulation prices $a$ and $b$ and the EST parameters. The sketch of the proof is provided in the appendix. 


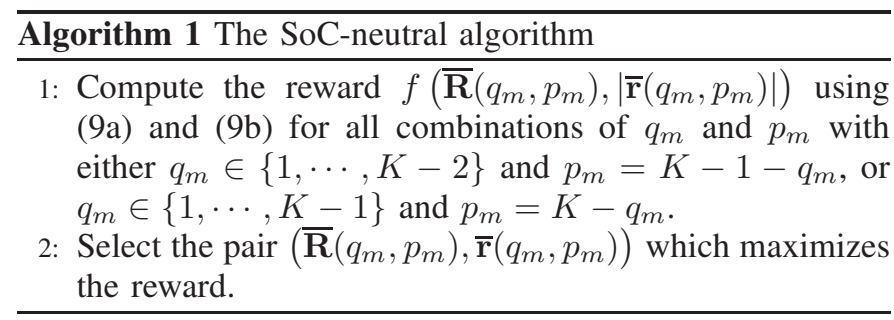

Lemma 2. Given $B, K, \delta, \Delta_{d}, \Delta_{c}, e_{d}, e_{c}, a$, and $b$, let $\mathcal{U}^{\star}$ denote the set of all initial SoC for which the SRU obtains maximum reward. Then, we have: $\frac{B}{2} \in \mathcal{U}^{\star}$.

\section{B. Multiple Contracts}

Let us assume that the SRU bids for a set of $N$ consecutive contracts of total duration $T=N D$, and that $U_{1}=B / 2$ denotes the $\mathrm{SoC}$ at the beginning of the first contract. The SRU can bid for multiple contracts without any concern for the SoC at the end of each contract since the operator provides regulation signals that keep the SoC at the end of each contract to be $U_{1}$. Hence, given $a_{n}, b_{n}$, and $U_{n}=B / 2$ for all $n \in\{1, \cdots, N\}$, the SRU can compute the reward $F_{T}=\sum_{n=1}^{N} f_{n}\left(\overline{\mathbf{R}}_{n},\left|\overline{\mathbf{r}}_{n}\right|\right)$ that can be obtained over the $N$ contracts. Next, we focus on the energy-neutral framework.

\section{ENERGY-NeUtral FramewORK}

In the energy-neutral contractual framework, the operator commits to generate a sequence of regulation signals $\left\{s_{k}\right\}$ so that the energy supplied by the SRU is equal to the energy absorbed by the SRU, i.e., the regulation signals obey the following constraints:

$$
\sum_{k=1}^{K}\left[s_{k}\right]^{+}=\sum_{k=1}^{K}\left[-s_{k}\right]^{+}
$$

The SRU can respond to any sequence of regulation signals $\left\{s_{k}\right\}$ if and only if the constraints (5)-(7) are satisfied. The SRU knows that the regulation signals obey (4) and (10). Using this information, the SRU selects its parameters $R$ and $r$ so that it can respond to all possible sequences of regulation signals $\left\{s_{k}\right\}$ without any failure, and his reward $f(R,|r|)$ is maximized. We first compute the values of $R$ and $r$ that maximize the reward for one single contract of duration $D=K \delta$, given the initial $\operatorname{SoC} U$. Then, we focus on $N$ successive contracts, and quantify the reward that can be obtained over the $N$ contracts.

\section{A. Single contract}

The arguments for the single contract case in the energyneutral contract follow the same lines as those for the SoCneutral contract, and are therefore not repeated here for the sake of brevity. The sequence of regulation signals, $\pi_{m}$, that results in the highest SoC level starts with $q_{m}$ time-slots of regulation signal $r$, where

$$
q_{m}=\left\lceil\frac{(K-1) R+r}{(|r|+R)}\right\rceil
$$

while the last $p_{m}=\left(K-1-q_{m}\right)$ time-slots have regulation signal $R$. There is a single time-slot in between the two, i.e., the $\left(q_{m}+1\right)^{t h}$ time-slot, with regulation signal $\left(-w_{m}\right)\left(\frac{1}{e_{c}} \mathbf{1}_{\left\{w_{m}>0\right\}}+\frac{1}{e_{d}} \mathbf{1}_{\left\{w_{m} \leq 0\right\}}\right)$ where $w_{m}$ is equal to $\left(e_{d} p_{m} R+e_{c} q_{m} r\right)$.

Lemma 3. Given $e_{c}, e_{d}, \Delta_{c}, \Delta_{d}, U, \delta, B$, the reward function $f(R,|r|)$, and with $q_{m} \in\{1, \cdots, K-2\}$ and $p_{m}=K-1-$ $q_{m}$, or $q_{m} \in\{1, \cdots, K-1\}$ and $p_{m}=K-q_{m}$, the pair $(\overline{\mathbf{R}}, \overline{\mathbf{r}})$ that maximizes the reward, can be computed by

$$
\begin{aligned}
& \widehat{\mathbf{R}}\left(q_{m}, p_{m}\right) \triangleq \min \left\{\Delta_{d}, \frac{q_{m}}{p_{m}} \Delta_{c}, \frac{B-U}{e_{c} \delta p_{m}}, \frac{U}{e_{d} \delta p_{m}}\right\} \\
& \left|\widehat{\mathbf{r}}\left(q_{m}, p_{m}\right)\right| \triangleq \min \left\{\Delta_{c}, \frac{p_{m}}{q_{m}} \Delta_{d}, \frac{B-U}{e_{c} \delta q_{m}}, \frac{U}{e_{d} \delta q_{m}}\right\} .
\end{aligned}
$$

A formal description of the proposed algorithm to compute the optimal values of $R$ and $r$ is provided in Algorithm 2 .

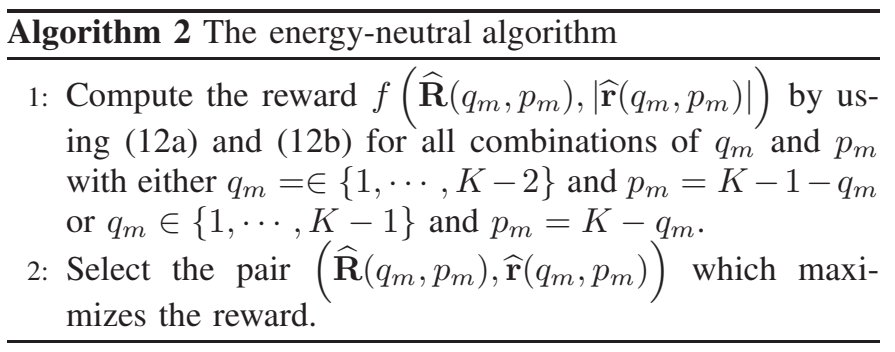

Our analytical results in (12a) and (12b) show that the optimal values of the regulation parameters $R$ and $r$ depend on the value of the initial SoC $U$. We can verify that the value of the initial SoC $U^{\star}$ for which the SRU obtains the highest possible reward, is equal to $B /\left(1+\frac{e_{c}}{e_{d}}\right)$.

\section{B. Multiple contracts}

Let us assume that the SRU bids for a set of $N$ consecutive contracts of total duration $T=N D$, and that the SoC at the beginning of the first contract is fixed and known. The reward in contract $C_{n}$ is a function of the parameters $\widehat{\mathbf{R}}_{n}$ and $\widehat{\mathbf{r}}_{n}$ which are functions of the $\mathrm{SoC}$ at the beginning of the contract (we call it $U_{n}$ ). Since $U_{1}$ is fixed and known, $\widehat{\mathbf{R}}_{1}$ and $\widehat{\mathbf{r}}_{1}$ can be computed by using Algorithm 2 . The value of $U_{n}$ for $n>1$ depends on the sequences of regulation signals being produced by the operator during contracts $C_{1}, \cdots, C_{n-1}$. Since the sequences that can be sent by the operator, are unknown a priori, the initial SoC $U_{n}$ as well as the reward $f_{n}\left(\widehat{\mathbf{R}}_{n},\left|\widehat{\mathbf{r}}_{n}\right|\right)$ are random variables. We characterize a priori the reward $F_{T}=\sum_{n=1}^{N} f_{n}\left(\widehat{\mathbf{R}}_{n},\left|\widehat{\mathbf{r}}_{n}\right|\right)$ that the SRU can obtain over the $N$ contracts, by providing upper and lower bounds on $F_{T}$.

Upper and Lower Bounds: Let us begin with a single contract $n$, and assume that we do not know the initial SoC $U_{n}$ at the beginning of that contract. We compute upper and lower bounds on the reward that can be obtained in contract $C_{n}$ in two steps. In the first step, we find upper and lower bounds on the $\mathrm{SoC}$ at the beginning of contract $C_{n}$. In the second step, we compute the maximum and minimum reward that can be obtained by the SRU, knowing that the initial SoC $U_{n}$ is in the range $\left[L b_{n}, U b_{n}\right]$ where $U b_{n}$ and $L b_{n}$ denote the computed upper and lower bounds on $U_{n}$, respectively.

To compute upper and lower bound on $U_{n}$, let $E_{n}$ denote the amount of energy supplied by the SRU in contract $C_{n}$, i.e., $\sum_{k=1}^{K}\left[s_{k}\right]^{+}$. In the energy-neutral framework, the amount 
of energy absorbed by the SRU in one single contract is equal to the amount of energy supplied by the SRU in the contract. Therefore, the storage supplies (resp. draws) $e_{d} E_{n}$ (resp. $e_{c} E_{n}$ ) units of energy in contract $C_{n}$. Hence, the SoC at the end of contract $n$ is equal to $U_{n-1}+\left(e_{c}-e_{d}\right) E_{n-1}$. Since the charging efficiency $e_{c}$ is less than or equal to the discharging efficiency $e_{d}$, we can verify that $U_{n} \in\left[U_{n-1}+\right.$ $\left.\left(e_{c}-e_{d}\right) \widehat{E}_{n-1}, U_{n-1}\right]$ where $\widehat{E}_{n-1}$ is an upper bound on the maximum amount of energy which can be supplied by the SRU in contract $C_{n-1}$ given the initial SoC $U_{n-1}$. Using this observation, we can verify that $U b_{n}=U_{1}$ for all $n$.

Using the result in Lemma 3 , we find that we can take $\widehat{E}_{n-1}$ to be equal to the minimum of $\frac{U_{n-1}}{e_{d}}$ and $\left(B-U_{n-1}\right) / e_{c}$. We use this property as the basis of an algorithm to compute iteratively the value of $L b_{n}$ for all $n$. At each iteration $n$, we assume that the initial SoC $U_{n}$ is equal to $L b_{n}$, and compute the values of $\widehat{\mathbf{R}}_{n}, \widehat{\mathbf{r}}_{n}$, and $\widehat{E}_{n}$. We then take $L b_{n+1}$ to be equal to $L b_{n}+\left(e_{c}-e_{d}\right) \widehat{E}_{n}$. The following result shows that $L b_{n}+$ $\left(e_{c}-e_{d}\right) \widehat{E}_{n}$ is a lower bound on the initial SoC in contract $C_{n+1}$. A sketch of the proof is provided in the appendix.

Lemma 4. Given $B, e_{c}, e_{d}$, and $L b_{n}$, let $\widehat{E}_{n}=$ $\min \left\{\left(B-L b_{n}\right) / e_{c},\left(L b_{n} / e_{d}\right)\right\}$. Then, $L b_{n}+\left(e_{c}-e_{d}\right) \widehat{E}_{n}$ is a lower bound on the initial SoC in contract $C_{n+1}$.

In the proposed algorithm, we first fix $L b_{1}=U_{1}$, and compute the values of the parameters $\widehat{\mathbf{R}}_{1}, \widehat{\mathbf{r}}_{1}$, and $\widehat{E}_{1}$. We then take the value of $L b_{2}$ to be equal to $\left(U_{1}+\left(e_{c}-e_{d}\right) \widehat{E}_{1}\right)$, and compute $\widehat{\mathbf{R}}_{2}, \widehat{\mathbf{r}}_{2}$, and $\widehat{E}_{2}$ assuming that $U_{2}=L b_{2}$. By doing the same process for $C_{3}, \cdots, C_{n}$, we can compute the lower bound $L b_{n}$ for all the $N$ contracts. A formal description of the algorithm is provided in Algorithm 3.

To compute upper and lower bounds on $F_{T}$, let $\overline{f_{n}}$ and $\underline{f_{n}}$ denote the maximum and minimum values of $f_{n}\left(\widehat{R}_{n},\left|\widehat{r}_{n}\right|\right)$ over $\left[L b_{n}, U_{1}\right]$, respectively. Proposition 1 derives the values of $U$ at which $\overline{f_{n}}$ and $f_{n}$ are attained. The proof follows by using Lemma 3. The lemma shows that the minimum of $\frac{U}{e_{d}}$ and $(B-U) / e_{c}$ is one of the parameters that determine the reward, and that the values of $U$ at which the maximum and minimum values $\overline{f_{n}}$ and $f_{n}$, respectively, are achieved, do not depend on the prices $a_{n}$ and $b_{n}$. Due to space limitations, we do not present the proof.

Proposition 1. Let $a_{n} \geq 0$ and $b_{n} \geq 0$ denote the prices for each unit of upward and downward regulation in contract $n$. Given $e_{c}, e_{d}, B, U_{1}$, and $L b_{n}$, the maximum and minimum values of $f_{n}\left(\widehat{\mathbf{R}}_{n},\left|\widehat{\mathbf{r}}_{n}\right|\right)$ over $\left[L b_{n}, U_{1}\right]$ are attained at $\overline{U_{n}}$ and $U_{n}$, respectively, where

Case 1) If $U_{1} \leq \frac{e_{d} B}{e_{d}+e_{c}}$, then

$$
\underline{U_{n}}=L b_{n}, \quad \overline{U_{n}}=U_{1} .
$$

Case 2) If $U_{1}>\frac{e_{d} B}{e_{d}+e_{c}}$ and $L b_{n} \geq \frac{e_{d} B}{e_{d}+e_{c}}$, then

$$
\underline{U_{n}}=U_{1}, \quad \overline{U_{n}}=L b_{n} .
$$

Case 3) If $U_{1}>\frac{e_{d} B}{e_{d}+e_{c}}, L b_{n}<\frac{e_{d} B}{e_{d}+e_{c}}$, and $\frac{B-U_{1}}{e_{c}}<\frac{L b_{n}}{e_{d}}$, then

$$
\underline{U_{n}}=U_{1}, \quad \overline{U_{n}}=\frac{e_{d} B}{e_{d}+e_{c}} .
$$

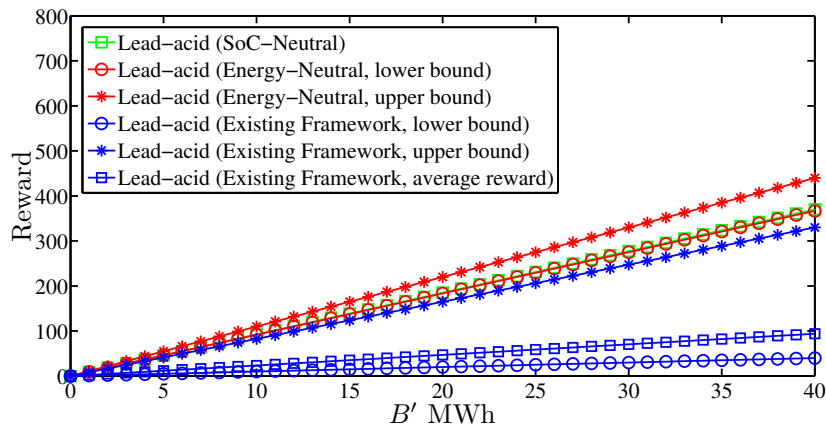

Fig. 1. Lead-acid: The lower and upper bounds on $F_{T}$, as a function of $B^{\prime}$ for $D=1 h$ and $U_{1}=B / 2$.

TABLE I

STORAGE CHARACTERISTICS [10], [11]

\begin{tabular}{|c|c|c|}
\hline Storage technology & Lead-acid & Lithium-ion \\
\hline \hline Charging efficiency $e_{c}$ & 0.75 & 0.85 \\
\hline Discharging efficiency $e_{d}$ & 1 & 1 \\
\hline Charge time $\alpha$ & $10 \mathrm{~h}$ & $3 \mathrm{~h}$ \\
\hline Discharge time $\gamma$ & $1 \mathrm{~h}$ & $36 \mathrm{~min}$ \\
\hline DoD $\eta$ & 0.8 & 0.8 \\
\hline
\end{tabular}

Case 4) If $U_{1}>\frac{e_{d} B}{e_{d}+e_{c}}, L b_{n}<\frac{e_{d} B}{e_{d}+e_{c}}$, and $\frac{B-U_{1}}{e_{c}} \geq \frac{L b_{n}}{e_{d}}$, then

$$
\underline{U_{n}}=L b_{n}, \quad \overline{U_{n}}=\frac{e_{d} B}{e_{d}+e_{c}} .
$$

Given $a_{n}, b_{n}$, and $\underline{U_{n}}$ (resp. $\overline{U_{n}}$ ), we can compute the lower bound $f_{n}$ (resp. the upper bound $\overline{f_{n}}$ ) by computing the value of $f_{n}\left(\widehat{\mathbf{R}}_{n},\left|\widehat{\mathbf{r}}_{n}\right|\right)$ at $U=U_{n}$ (resp. at $\left.U=\overline{U_{n}}\right)$. The reward $F_{T}$ over the $N$ contracts is then bounded as follows:

$$
\sum_{n=1}^{N} \underline{f_{n}} \leq F_{T} \leq \sum_{n=1}^{N} \overline{f_{n}}
$$

Note that in the energy-neutral framework, the battery will eventually discharge since $U b_{n}=U_{1}$ and $L b_{n+1}=L b_{n}+$ $\left(e_{c}-e_{d}\right) \widehat{E}_{n}$ with $\left(e_{c}-e_{d}\right) \leq 0$ and $\widehat{E}_{n} \geq 0$. Therefore, after a limited number of contracts, the SRU will not be able to participate in an energy-neutral contract. The SRU can either purchase electricity from the market or participate in a pure downward regulation service to charge its battery.

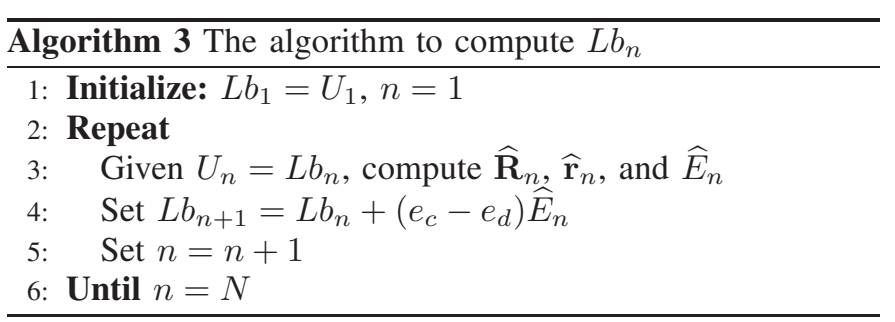

In the next section, we provide engineering insights based on our analytical results, i.e., the proposed lower and upper bounds on $F_{T}$ in the energy-neutral framework, and the exact value of the reward $F_{T}$ in the SoC-neutral framework. 


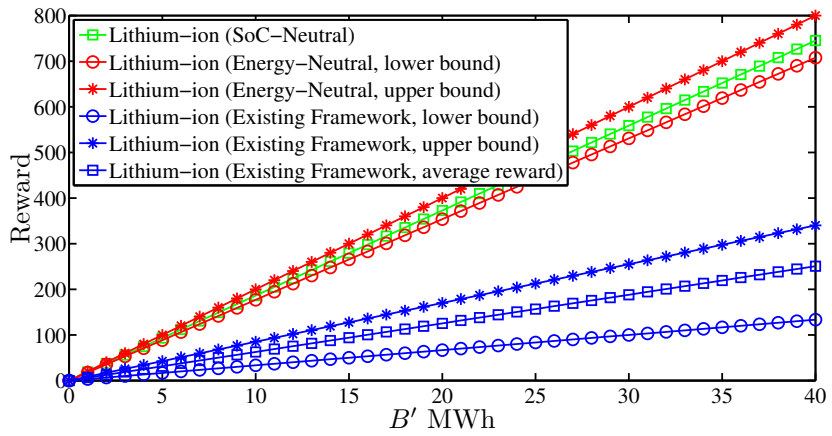

Fig. 2. Lithium-ion: The lower and upper bounds on $F_{T}$, as a function of $B^{\prime}$ for $D=1 h$ and $U_{1}=B / 2$.

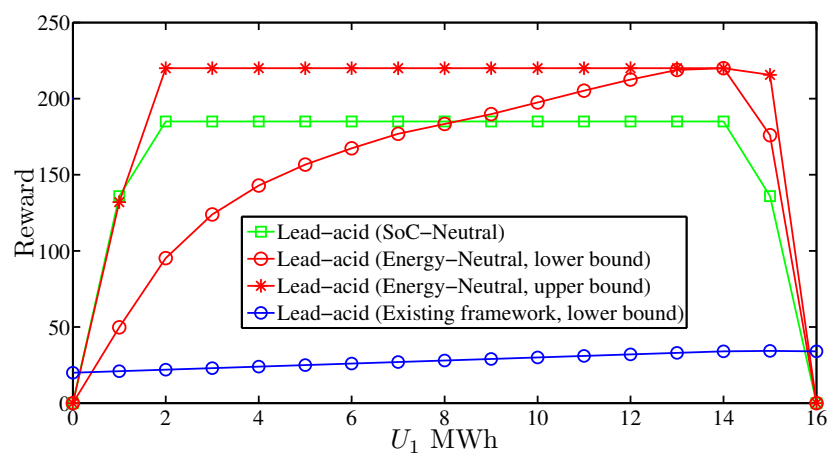

Fig. 3. Lead-acid: The lower and upper bounds on $F_{T}$, as a function of $U_{1}$ for $B^{\prime}=20 \mathrm{MWh}$.

\section{NuMERICAL RESULTS}

In this paper, we consider two of the battery technologies that have been considered in the literature as potential candidates to offer a regulation service, namely Lead-acid (LA) and Lithium-ion (LI). The depth-of-discharge (DoD) is given by $\eta$ and therefore the effective battery capacity, $B^{\prime}$ equals $\eta B$. To determine the maximum charging and discharging limits, $\Delta_{c}$ and $\Delta_{d}$ respectively, we use published data on the charge and discharge times, $\alpha$ and $\gamma$ respectively, for the two technologies. That is, $\Delta_{c}=B^{\prime} / \alpha$ and $\Delta_{d}=B^{\prime} / \gamma$. The battery technology parameters used in this study are summarized in Table I.

The number of time-slots in each contract is set to $K=$

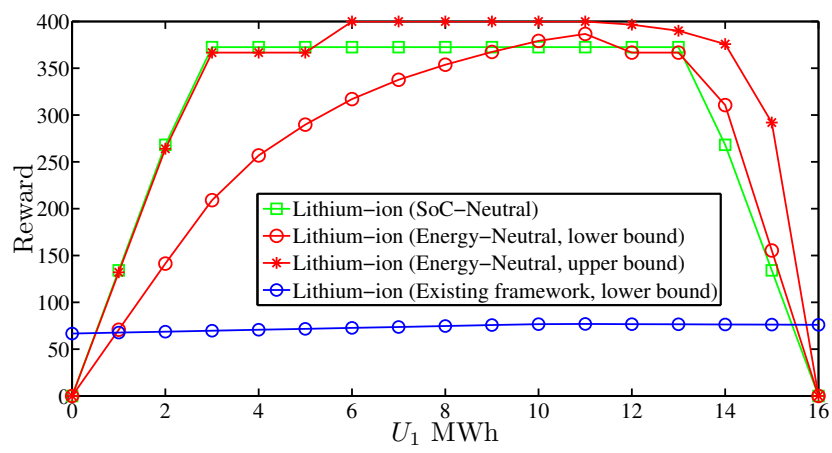

Fig. 4. Lithium-ion: The lower and upper bounds on $F_{T}$, as a function of $U_{1}$ for $B^{\prime}=20 \mathrm{MWh}$.

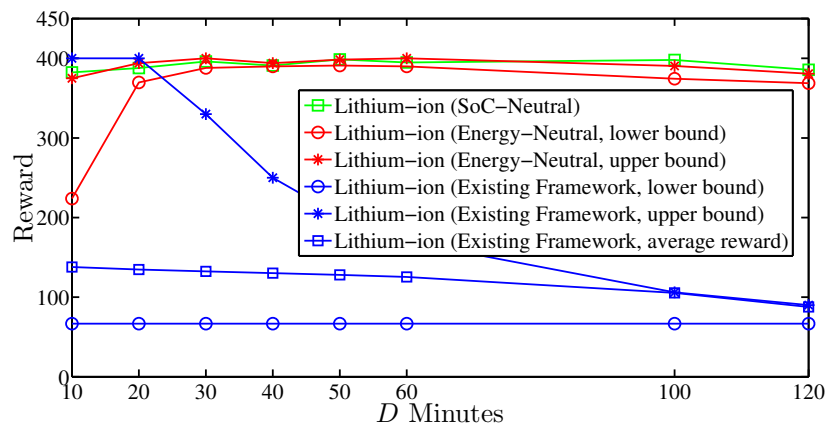

Fig. 5. Lithium-ion: The lower and upper bounds on $F_{T}$, as a function of $D$ for $B^{\prime}=20 \mathrm{MWh}, \delta=1 \mathrm{~min}$, and $U_{1}$ that maximizes the lower bound.

12 , the duration of a contract is assumed to be $D=1$ hour, such that each time-slot is $\delta=5$ minutes long. Rewards are computed over 10 successive contracts (i.e., $T=10$ hours) and $a_{n}=b_{n}=1 \$ /(\mathrm{h} \mathrm{MW})$. Finally, unless otherwise stated, the initial SoC at the beginning of the first contract is $U_{1}=B / 2$.

We compute the average reward as well as the lower and upper bounds on $F_{T}$ for the existing framework using [9], the lower and upper bounds proposed in Section V-B for the energy-neutral framework, and the exact value of the reward $F_{T}$ for the SoC-neutral framework. Figures 1-2 compare the three contractual frameworks in terms of the minimum and maximum reward as a function of battery capacity, for LA and LI, respectively. Our numerical results show that the minimum achievable reward with the SoC-neutral and energy-neutral frameworks is significantly larger than the maximum achievable reward by the existing framework, i.e., from the SRU standpoint, the SoC-neutral and the energy-neutral frameworks are significantly more beneficial than the existing framework.

The results also show that the achievable reward with the SoC-neutral framework is between the minimum and maximum achievable rewards by the energy-neutral framework. We note, however, that if the number of contract durations over which reward is computed increases, we expect that the SoC neutral framework will provide strictly higher rewards since it enables us to maintain the SoC at the preferred level over successive contracts. Finally, we also observe that LI provides greater rewards than LA in all the three contractual frameworks for a given $B^{\prime}$. We have obtained similar results for different values of $D$ and $T$.

To understand the impact of the initial SoC charge $U_{1}$ on the SRU's reward $F_{T}$, we compute and plot the lower and upper bounds on $F_{T}$ as a function of $U_{1}$ for the existing and energyneutral frameworks, and the exact value of the reward $F_{T}$ for the SoC-neutral framework, for LA and LI in Fig. 3 and Fig. 4, respectively. We observe that for both battery technologies, the reward in the energy-neutral contract has greater sensitivity to the choice of $U_{1}$ than in the SoC-neutral contract. Thus, although operators might prefer energy-neutral contracts since it obviates the need for the operator to consider the technology parameters of the EST used by each SRU, SRUs might prefer SoC-neutral contracts for their predictability.

The SRU could also influence the operator to negotiate a contract duration which is favorable to its storage technology. 
To understand the impact of varying the contract duration $D$ (equivalently, varying $K$ with $\delta$ fixed) on the SRU's reward in different frameworks, we consider a period of length 10 hours (i.e., $T=10 \mathrm{~h}$ ), and take $B=20 \mathrm{MWh}$ and $\delta=1$ minute. The results for LI are shown in Fig. 5 (results for LA are similar and thus omitted due to space constraints). Our numerical results show that while reward decreases for longer contracts with the existing framework, the opposite is true for the energy- and SoC-neutral frameworks. The results show that beyond a contract duration of 30 minutes, the reward for both the SoC-and energy-neutral contracts are relatively insensitive to $D$, while this is not the case for the existing framework.

\section{CONCLUSION}

This paper provides tools to quantify offline the reward an SRU could expect for the energy-neutral contractual framework as well as for the SoC-neutral framework. This paper takes the standpoint of the SRU. We plan to address the operator's standpoint in a subsequent paper.

\section{APPENDIX}

Although we cannot provide complete proofs due to space constraints, we provide below sketches of our proofs.

Proof of Lemma 1: Let us assume that $p_{m}=K-q_{m}$. Given $q_{m}$ and $p_{m}$, we can construct the worst-case sequence $\pi_{m}$ that can be sent by the operator. Given an initial SoC $U$, a pair $(R, r)$ with the worst-case sequence $\pi_{m}$ can be chosen by the SRU if the value of the SoC at this sequence is in the range $[0, B]$. Let $\bar{s}_{m}$ denote the $\left(q_{m}+1\right)^{t h}$ signal, i.e., $\bar{s}_{m}=$ $\left(-w_{m}\right)\left(\frac{1}{e_{c}} \mathbf{1}_{\left\{w_{m}>0\right\}}+\frac{1}{e_{d}} \mathbf{1}_{\left\{w_{m} \leq 0\right\}}\right)$ where $w_{m}=\left(e_{d} p_{m} R+\right.$ $\left.e_{c} q_{m} r\right)$. To find the pair $(R, r)$ that maximizes the reward function $f(R,|r|)$, we consider the following cases:

1) Pairs $(R, r)$ for which $0 \leq \bar{s}_{m} \leq R$ : the SoC will be in the range $[0, B]$ iff $\frac{e_{c} q_{m}}{e_{d}\left(p_{m}+1\right)}|r| \leq R$ and $R \leq \frac{e_{c} q_{m}}{e_{d} p_{m}}|r|$.

2) Pairs $(R, r)$ for which $r \leq \bar{s}_{m} \leq 0$ : the SoC will be in the range $[0, B]$ iff $\frac{e_{c} q_{m}}{e_{d} p_{m}}|r| \leq R$ and $R \leq \frac{e_{c}\left(q_{m}+1\right)}{e_{d} p_{m}}|r|$.

Therefore, the only possible scenario is to have $\bar{s}_{m} \geq 0$.

Let us focus on pairs $(R, r)$ for which $0 \leq \bar{s}_{m} \leq R$. Using the definition of $\bar{s}_{m}$, we can show that the SoC will be in the range $[0, B]$ iff $|r| \leq \frac{\min \{B-U, U\}}{e_{c} \delta q_{m}}$. We also need to make sure that $|r| \leq \Delta_{c}$. Therefore, the pair $\left(R_{1}, r_{1}\right)$ where $\left|r_{1}\right|=\left|r_{0}\right|, R_{1}=\frac{e_{c} q_{m}}{e_{d}\left(p_{m}+1\right)}\left|r_{1}\right|$, and $\left|r_{0}\right|=$ $\min \left\{\Delta_{c}, \frac{B-U}{e_{c} \delta q_{m}}, \frac{U}{e_{c} \delta q_{m}}\right\}$, maximizes the reward among all feasible pair for which $0 \leq \bar{s}_{m} \leq R$. Similarly, we can show that the pair $\left(R_{2}, r_{2}\right)$ where $R_{2}=R_{0},\left|r_{2}\right|=\frac{e_{d} p_{M}}{e_{c}\left(q_{M}+1\right)} R_{0}$, and $R_{0}=\min \left\{\Delta_{d}, \frac{B-U}{e_{d} \delta p_{M}}, \frac{U}{e_{d} \delta p_{M}}\right\}$, maximizes the reward among all feasible pairs for which $r \leq \bar{s}_{M} \leq 0$. Note that the pairs $\left(R_{1}, r_{1}\right)$ and $\left(R_{2},\left|r_{2}\right|\right)$ do not necessarily satisfy $R \leq \Delta_{d}$ and $|r| \leq \Delta_{c}$, respectively. The optimal pair at which all the physical constraints are satisfied is $(\overline{\mathbf{R}},|\overline{\mathbf{r}}|)$ where $\overline{\mathbf{R}}=\min \left(R_{1}, R_{2}\right)$ and $\min \left(\left|r_{1}\right|,\left|r_{2}\right|\right)$. The case at which $p_{m}=K-1-q_{m}$ can be proved by following the same argument as above.

Proof of Lemma 2: Let us assume that $\frac{B}{2} \notin \mathcal{U}^{\star}$. Hence, there exists $U_{0} \in \mathcal{U}^{\star}\left(U_{0} \neq \frac{B}{2}\right)$ for which there exist pair $\left(R_{0}, r_{0}\right)$ and worst-case sequences $\pi_{M}$ and $\pi_{m}$ such that the value of the $\mathrm{SoC}$ for these sequences is in the range $[0, B]$ and the reward is equal to $z^{\star}$. Since all feasible sequences $\pi$ for the pair $\left(R_{0}, r_{0}\right)$ obey (3), the maximum charging (or discharging) energy in one contract is less than or equal to $\frac{B}{2}$. Therefore, the initial SoC $\frac{B}{2} \in \mathcal{U}^{\star}$. This results in a contradiction.

Proof of Lemma 4: Claim: For any two values of $U_{a}, U_{b} \in$ $\left[L b_{n}, U_{1}\right]$ with $U_{a} \geq U_{b}$, we have:

$$
U_{a}+\left(e_{c}-e_{d}\right) \widehat{E}_{n}\left(U_{a}\right) \geq U_{b}+\left(e_{c}-e_{d}\right) \widehat{E}_{n}\left(U_{b}\right)
$$

where $\widehat{E}_{n}(U)=\min \left\{(B-U) / e_{c},\left(U / e_{d}\right)\right\}$ is an upper bound on the maximum amount of energy that can be supplied by the SRU in contract $C_{n}$ when the initial SoC equals $U$.

Proof : Let us consider the following three cases: 1) $U_{b} \leq$ $U^{\star} \leq U_{a}$, 2) $U_{b} \leq U_{a} \leq U^{\star}$, and 3) $U^{\star} \leq U_{b} \leq U_{a}$, where $U^{\star}=\frac{e_{d} B}{e_{c}+e_{d}}$. Without loss of generalities, let us assume that $U_{b}=U_{a}-x$ where $U_{a}-U^{\star} \leq x \leq U_{a}$. Let us focus on the first case, and define $P\left(U_{a}, x\right)$ as follows:

$$
\begin{aligned}
P\left(U_{a}, x\right) & \triangleq\left(U_{a}+\left(e_{c}-e_{d}\right) \widehat{E}_{n}\left(U_{a}\right)\right) \\
& -\left(\left(U_{a}-x\right)+\left(e_{c}-e_{d}\right) \widehat{E}_{n}\left(U_{a}-x\right)\right)
\end{aligned}
$$

By using the definition of $\widehat{E}_{n}(U)$, we can verify that

$$
P\left(U_{a}, x\right)=B\left(1-\frac{e_{d}}{e_{c}}\right)+U_{a}\left(\frac{e_{d}}{e_{c}}-\frac{e_{c}}{e_{d}}\right)+x \frac{e_{c}}{e_{d}}
$$

where $U^{\star} \leq U_{a} \leq B$ and $U_{a}-U^{\star} \leq x \leq U_{a}$. We need to show that $P \geq 0$ for all possible values of $U_{a}$ and $x$. Since $P$ is increasing in $x$, the minimum of $P$ is attained at $x=U_{a}-U^{\star}$. Since $e_{d} \geq e_{c}, P\left(U_{a}, U_{a}-U^{\star}\right)$ is increasing in $U_{a}$. Therefore, the minimum of $P$ is attained at $U_{a}=U^{\star}$ and $x=0$. Since $P\left(U^{\star}, 0\right)=0$, we have $P \geq 0$ for all $U_{a}, U_{b} \in\left[L b_{n}, U_{1}\right]$ with $U_{b} \leq U^{\star} \leq U_{a}$. We can prove the result for the other two cases by following the same argument. This completes the proof of the claim. The main result now follows by using the claim above, and the fact that $U_{n} \in\left[L b_{n}, U_{1}\right]$.

\section{REFERENCES}

[1] Y. V. Makarov, J. Ma, S. Lu, and T. B. Nguyen, "Assessing the Value of Regulation Resources Based on Their Time Response Characteristics", PNNL-17632, Pacific Northwest National Laboratory, June 2008.

[2] Available at www.ercot.com/content/gridinfo/etts/keydocs/ERCOT_ Storage_Issues_Whitepaper.doc.

[3] V. Pandurangan, H. Zareipour, and O. Malik, "Frequency regulation services: A comparative study of select North American and European reserve markets," NAPS, pp. 1-8, Sept. 2012.

[4] S. K. Aditya and D. Das, "Battery energy storage for load frequency control of an interconnected power system," Elect. Power Syst. Res., vol. 58, no. 3, pp. 179-185, 2001.

[5] N. Lu, M.R. Weimar, Y.V. Makarov, J. Ma, and V.V. Viswanathan, "The Wide-Area Energy Storage and Management System Battery Storage Evaluation,' PNNL-18679, Pacific Northwest National Laboratory, 2009.

[6] N. Lu, M.R. Weimar, Y.V. Makarov, F.J. Rudolph, S.N. Murthy, J. Arseneaux, and C. Loutan, "Evaluation of the flywheel potential for providing regulation service in California," in Proc. of Power and Energy Society General Meeting, 2010.

[7] Available at http://www.nyiso.com/public/webdocs/markets_operations/ committees/bic_miwg/meeting_materials/2010-01-21/Energy_Storage_ Resources.pdf.

[8] www.caiso.com/Documents/BeaconComments-RewewablesIntegrationMarket_ProductReviewPhase2Vision_Roadmap.pdf.

[9] D. Fooladivanda, C. Rosenberg, and S. Garg, "An Analysis of Energy Storage and Regulation," in Procc. of IEEE SmartGridComm, Nov. 2014.

[10] D. Wang, C. Ren, A. Sivasubramaniam, B. Urgaonkar, and H. Fathy, "Energy storage in datacenters: what, where, and how much?," in Procc. of ACM sigmetrics/performance, pages 187-198, June 2012.

[11] "Electric Energy Storage Technology Options," Electric Power Research Institute (EPRI), Palo Alto, CA, 2010. 